\title{
With a new clip technique surgically inducing varicocele in Sprague-Dawley rats
}

Wen-bin Guo ${ }^{1 \dagger}$, Cheng Yang ${ }^{1 \dagger}$, Jun Bian ${ }^{1 \dagger}$, Hui Xia', Jian-kun Yang' ${ }^{1}$ Qi-zhao Zhou', Ming-kun Chen ${ }^{1}, K^{1}$ ang-yi Xue , Wan-song Zhang ${ }^{1}$, Peng Wang ${ }^{1}$, Xin $\mathrm{Li}^{2^{*}}$ and Cun-dong Liu ${ }^{1 *}$

\begin{abstract}
Background: We introduced and recreated a more consistent and effective experimental varicocele rat model by a new clip technique.

Methods: A total of 40 rats were numbered and randomly assigned to 5 groups of 8 each, including sham surgery (Group I), conventional (Group II) and clip groups with 0.7, 0.8, $0.9 \mathrm{~mm}$ gap widths, respectively (Group III, IV, V). All of the rats in each group were sacrificed at 8 weeks after initial surgery, and the rats forming out with less than $1 \mathrm{~mm}$ diameter of left spermatic vein or no presence of the pampiniform plexus dilation were excluded from the experimental groups. The left spermatic vein (LSV) diameter, testicular weight, left kidney weight to body weight coefficients, kidney and testicular histology were determined.

Results: The baseline mean diameter of the LSV in Group I, II and III was $0.22 \pm 0.02,0.23 \pm 0.02$ and $0.22 \pm 0.03 \mathrm{~mm}$, respectively $(P=0.7504)$. At 8 weeks after initial surgery, varicocele was successfully created in $6 / 8(75 \%), 7 / 8(87.5 \%)$, 3/8 (37.5\%), 3/8 (37.5\%) in Groupll-V, no varicocele was observed in Group I. In Group I, II and III, no pathological changes were observed and the left kidney weight to body weight coefficients showed no significant differences. The diameter of LSV was remarkably increased both in Group II and III compared to Group I $(1.72 \pm 0.13,1.57 \pm 0.19$ and $0.25 \pm 0.02$, respectively), and Group II and III had a smaller testicular weight than the rats in Group I (1.67 \pm 0.05 , $1.62 \pm 0.06$, and $1.92 \pm 0.12$, respectively).

Conclusions: With a new clip technique, surgically inducing varicocele rat model becomes convenient and safe. This appears to improve the effectiveness of the model and this innovation may allow us to further understand the pathophysiology of varicocele.
\end{abstract}

Keywords: Clip, Varicocele, Spermatic vein, Rat model

\section{Background}

Varicocele (VC) is a condition characterized by the spermatic varicosity or abnormal dilation of testicular pampiniform venous plexus, and is present in 35\% of men with primary infertility and in 70 to $81 \%$ with secondary infertility [1, 2]. Although it has been considered to be associated with male infertility, the exact pathophysiology of the varicocele is incompletely understood [3].

\footnotetext{
* Correspondence: xinli268@gmail.com; cundongliu@163.com

tWen-bin Guo, Cheng Yang and Jun Bian contributed equally to this work. ${ }^{2}$ Center for Clinical Research and Innovation, Shenzhen Hospital of Southern Medical University, Shenzhen, People's Republic of China

${ }^{1}$ Department of Urology, the Third Affiliated Hospital of Southern Medical University, No. 183 West Zhongshan Road, Tianhe District, 510630

Guangzhou, People's Republic of China
}

Specific questions regarding the pathophysiological of varicocele are even difficult to address in human. Several factors are often taken into account, such as limitedly acquisition of tissue, forbidden invasive experiment, or other indefinite characteristics [4]. Thus, this highlights the key role in the use of animal models. To date, the most widely used experimental varicocele rat model is to investigate the mechanisms underlying the varicocelerelated male infertility. However, experimental rat model induced by the conventional technique has varied success [5-7]. In the present study, for the first time we used a clip technique mimicking the nutcracker phenomenon suffering a partial occlusion of the renal vein combined with ligation of the branches of the LSV to develop

(c) The Author(s). 2018 Open Access This article is distributed under the terms of the Creative Commons Attribution 4.0 International License (http://creativecommons.org/licenses/by/4.0/), which permits unrestricted use, distribution, and 
experimental rat varicocele model, which was more consistent and effective to help to create varicocele rat model.

\section{Methods}

Animal care

This study was approved by the animal care and ethics committee of the Southern Medical University. A total of 40 adult male specific-pathogen free (SPF) Sprague Dawley rats (Rattus norvegicus, age range from 7 weeks to 8 weeks, weighing 250-300 g) obtained from the animal center of Southern Medical University (certificate number: SYXK [Yue] 2011-0074), were housed in a climate controlled environment with free access to food and water under a 12-h day/night cycle. A 1-week acclimatization period was allowed prior to the experimental procedures.

\section{Experimental design}

The 40 rats were numbered and randomly assigned to 5 groups of 8 each, with the method of random digits table, including sham surgery (Group I), conventional group (Group II), clip groups $(0.7 \mathrm{~mm}, 0.8 \mathrm{~mm}$ and $0.9 \mathrm{~mm}$ gap widths corresponding to Group III, IV, V, respectively) (obtained from ALCOTT BIOTECH, Shanghai, China). A sample size of 8 rats per group was sufficient according to our preliminary experiment and the previous study that the success rate of rat varicocele was ranged from 85 to $100 \%[8,9]$. At 8 weeks after initial surgery, all of the rats in each group were sacrificed. The postoperative outcomes were determined, including the left kidney histology, left kidney weight to body weight coefficients, the diameter of left spermatic vein (LSV), left testicular weight, histology and complications. The micrometer was used to measure the diameter of the LSV at the level of the crossing iliolumbar vein. All the operations were carried out by two skillful researchers, but they were not involved in the collection of data, analysis of results and evaluation of outcomes. All measurements were reviewed and based on agreement by 2 investigators independently. Other investigators and outcome assessors were blind to the groups.

\section{Experimental procedures}

In the present study, we created varicocele model in male Sprague-Dawley rats according to the procedures previously described by Turner TT [4], with partially occluding left renal vein and completely ligating the branches of the LSV. All experimental procedures were performed in a sterile environment. Briefly, after $12 \mathrm{~h}$ fasting, the rats were anesthetized using an intraperitoneal injection of $30 \mathrm{mg} / \mathrm{kg}$ sodium pentobarbital. The experimental rats were considered anaesthetized when holding and pressing the paw did not elicit a response from the rats. A midline laparotomy incision was made from xyphoid to pubis to visualize the left kidney, left adrenal vein, the left renal vein and the LSV and its collaterals. A tunnel was made around the left renal vein by careful blunt dissection, which was cleared of adhering tissue in a position medial to the insertion of the LSV and left adrenal vein. In Group II, a 4-0 silk suture was used for partially occluding of the left renal vein around a metal wire of $0.85 \mathrm{~mm}$ diameter at the point. The wire was then removed from the ligature, allowing the vein expand to an external diameter. In Group I, the rats with sham operation underwent a similar procedure that the left renal vein was dissected free but not ligated. In Group III, IV, and V, the clips with $0.7 \mathrm{~mm}, 0.8 \mathrm{~mm}$ and $0.9 \mathrm{~mm}$ gap widthsinstead of suture ligature was placed at the same point on the renal vein. Finally, the collaterals of the LSV were completely occluded and then the incision was closed in two layers with 3-0 silk sutures.

\section{Histopathology assessment}

After 8 weeks, the testes and kidneys were harvested and weighted. Half of each kidney and testis was fixed in $4 \%$ paraformaldehyde and modified Davidson's Fluid's solution [10], respectively. These were postfixed in $70 \%$ alcohol and embedded in paraffin blocks after tissue processing. Sections $(5 \mu \mathrm{m})$ from testes were obtained, deparaffinized and stained with hematoxylin and eosin. Disintegration of the germinal epithelium and degenerative appearance in each group was observed by light microscopy. For kidney histology, sections were stained with periodic acid-Schiff (PAS) and Masson's trichrome (Baso, Zhuhai, China). Histopathology analysis was performed by two uropathologists independently.

\section{Statistical analysis}

Statistical analysis was performed using SPSS 20, and all data are presented as the mean $\pm \mathrm{SD}$. Comparisons of all 3 groups and 2 groups were made using the KruskalWallis and the Games-Howell test for continuous variables, respectively. A value of $P<0.05$ was considered statistically significance.

\section{Results}

The characteristics of experimental varicocele development in each group

Some complications occurred in study. For example, Group II had 1 rat tearing of vein or bleeding and 1 rat pyonephrosis, which lead to model failure. In Group IV and $\mathrm{V}$, there were $3 / 8$, and $5 / 8$ clips showing slipping off the renal vein, respectively, while in Group III no severe complication happened and all rats had successful except for one rat failing to form varicocele. According to the criteria of $\mathrm{VC}$ model, the rats forming out with more than $1 \mathrm{~mm}$ diameter of left spermatic vein or presence of the pampiniform plexus dilation [4] in each group 
were included in the experimental groups [Fig. 1]. According to Table 1, we selected Group III for further analysis, excluding Groups IV and V because that the number of inducing varicocele is not enough to allow for extrapolations from the proposed statistics.

\section{Baseline anatomy and outcomes}

The mean baseline of total body weight of rats among Group I, II and III was $331.25 \pm 8.27,329.13 \pm 13.66$ and $326.75 \pm 12.49$ g, respectively $(P=0.6992)$. Baseline mean diameter of the left spermatic vein in these three groups was $0.22 \pm 0.02,0.23 \pm 0.02$ and $0.22 \pm 0.03 \mathrm{~mm}$, respectively $(P=0.7504)$. At 8 weeks after initial surgery, the size was remarkably increased when measuring the diameter of LSV both in Group II and III compared with Group I $(1.72 \pm 0.13,1.57 \pm 0.19$ and $0.25 \pm 0.02$, respectively, $P<0.0001$, Fig. 2a). No significant difference was observed between Group II and III $(P=0.275)$. Group II and III had smaller testicular weight compared with Group I $(1.67 \pm 0.05,1.62 \pm 0.06$, and $1.92 \pm 0.12$ respectively, $P<0.0001$, Fig. $2 b)$. There was also no difference between Group II and III $(P=0.196)$.

\section{Evaluation of experimental varicocele model}

After 8 weeks, macroscopic changes in the left kidney were carefully identified and compared with the right kidney. No pathological changes were observed in Group I, II and III [Fig. 3a]. The left kidney weight to body weight coefficients showed no significant differences among these three groups [Fig. 3b]. When calculating the numbers of degenerating tubules and compared the degenerating tubule percentages among these three groups, we found the percentage both in Group II and III were statistically significantly increased as compared with Group I [Fig. 4], which indicate impairment of spermatogenesis.
Table 1 The characteristics of experimental varicocele development in each group

\begin{tabular}{llllll}
\hline Group & Number & Survival rate & Complications & Varicocele & Success rate \\
\hline $\mathrm{I}^{\mathrm{a}}$ & 8 & $100 \%$ & 0 & 0 & 0 \\
$\|^{\mathrm{a}}$ & 8 & $100 \%$ & $3^{\mathrm{b}}$ & 6 & $75 \%$ \\
$\|^{\mathrm{a}}$ & 8 & $100 \%$ & 0 & 7 & $87.5 \%$ \\
$\mathrm{IV}^{\mathrm{a}}$ & 8 & $100 \%$ & 0 & 3 & $37.5 \%$ \\
$\mathrm{~V}^{\mathrm{a}}$ & 8 & $100 \%$ & 0 & 3 & $37.5 \%$ \\
\hline
\end{tabular}

I: Sham II: Conventional III: $0.7 \mathrm{~mm}$ clip IV: $0.8 \mathrm{~mm}$ clip V: $0.9 \mathrm{~mm}$ clip

${ }^{b_{2}} 2$ rats bleeding and 1 pyonephrosis

\section{Discussions}

Despite the fact that varicocele associated with male subfertility is widely accepted [11], the pathophysiology of varicocele has been poorly understood. With rare exception, varicocele does occur exclusively in humans, which are more difficult to address. Thus, the use of animal models has played an essential role in understanding this condition. In 1981, Saypol et al. [12] reported that surgically induced varicocele using partial left renal vein occlusion had influence on testicular blood flow, temperature, and histology in adult rats and dogs. Subsequently, many studies investigating on the pathophysiology underlying varicocele associated with infertility were conducted by this method [4, 13-15]. As similarities existed in venous anatomy between the rat and human when left varicocele occurs [16], since then experimental varicocele in rat has been the most widely used animal model. However, it has resulted in varied success except for interlaboratory and interoperator intrinsic variability $[6,7]$.

Previous studies on the variable vascular anatomy in rats may contribute to develop varicocele rat model $[5,17,18]$. In most rats, the left venous pampiniform plexus drains primarily into the left common iliac and a smaller internal spermatic vein to the left renal vein $[5,19]$. In the present study, we created varicocele rat

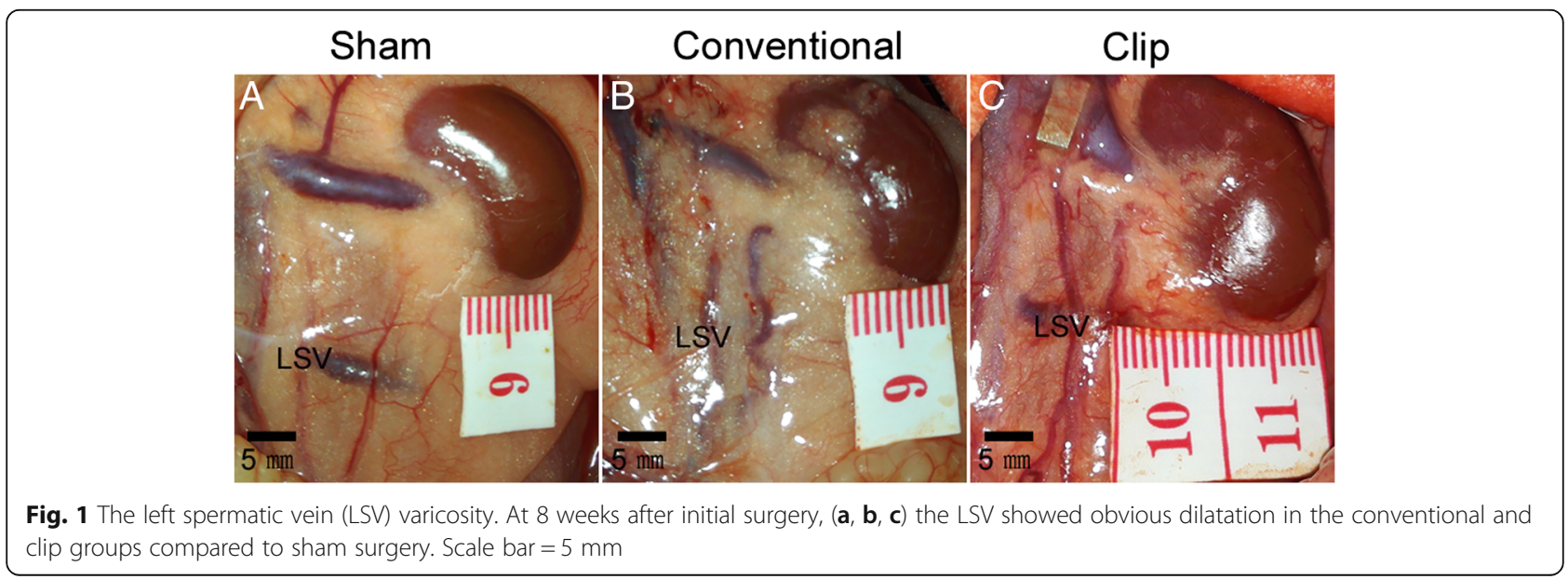



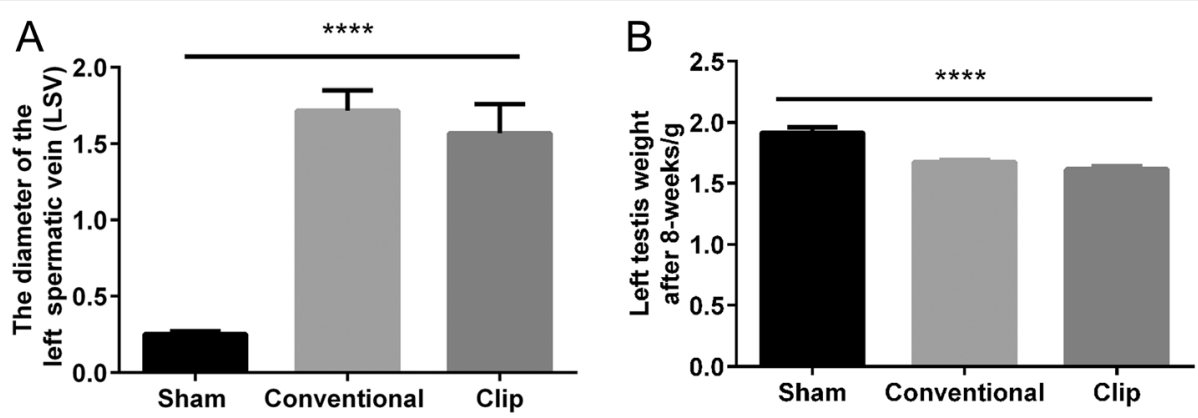

Fig. 2 The diameter of the left spermatic vein (LSV) a At 8 weeks after initial surgery, the diameter of LSV was remarkably larger and $\mathbf{b}$ the left testis weight was smaller in the conventional and clip groups when compared with sham surgery $(* * * P<0.0001)$

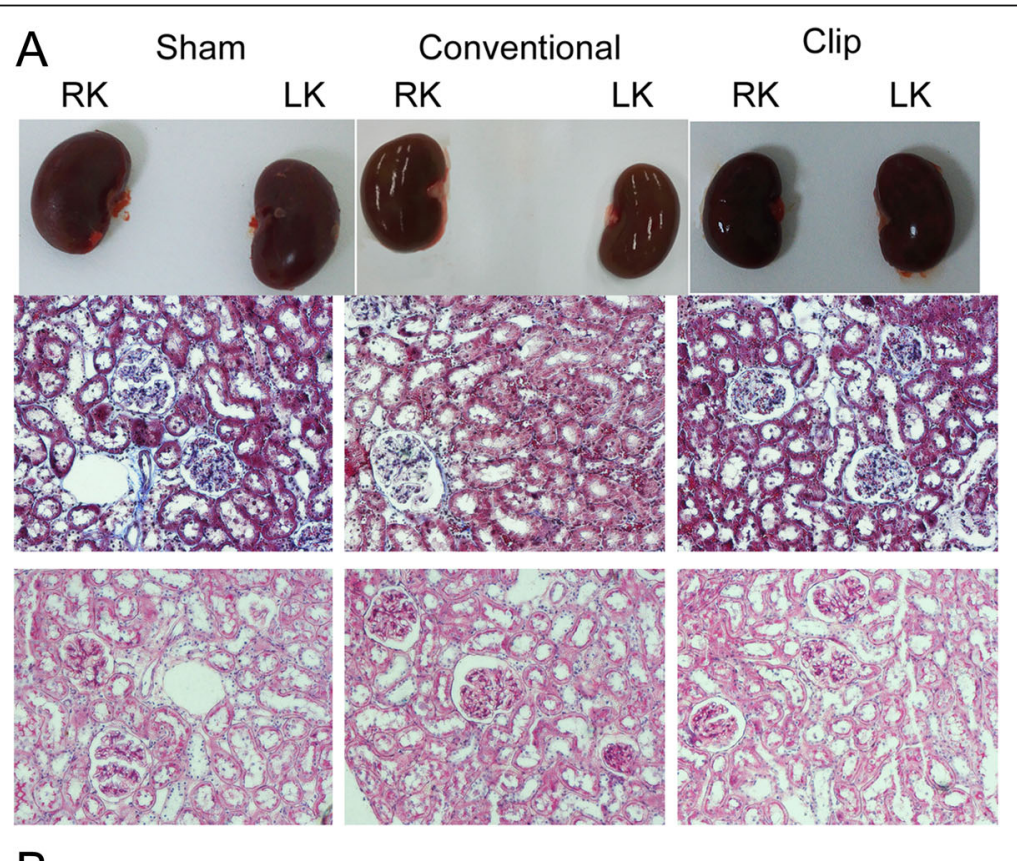

B

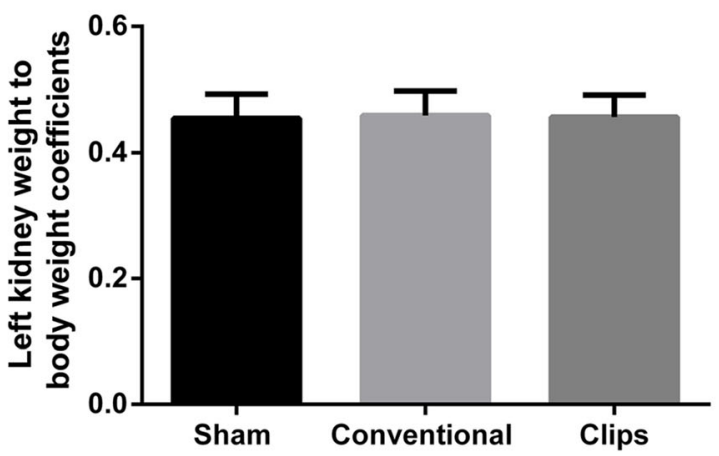

Fig. 3 Histopathology assessment of left kidney. (a) ( $a, b, c)$ Masson's Trichrome staining and ( $d, e$, f) PAS staining of the left kidney showed no significantly pathological changes in the sham, conventional and clip groups after 8 weeks. Scale bar $=50 \mu m$. Right kidney: RK, Left kidney: LK. (b) The left kidney weight to body weight coefficients showed no significant differences among these three groups 


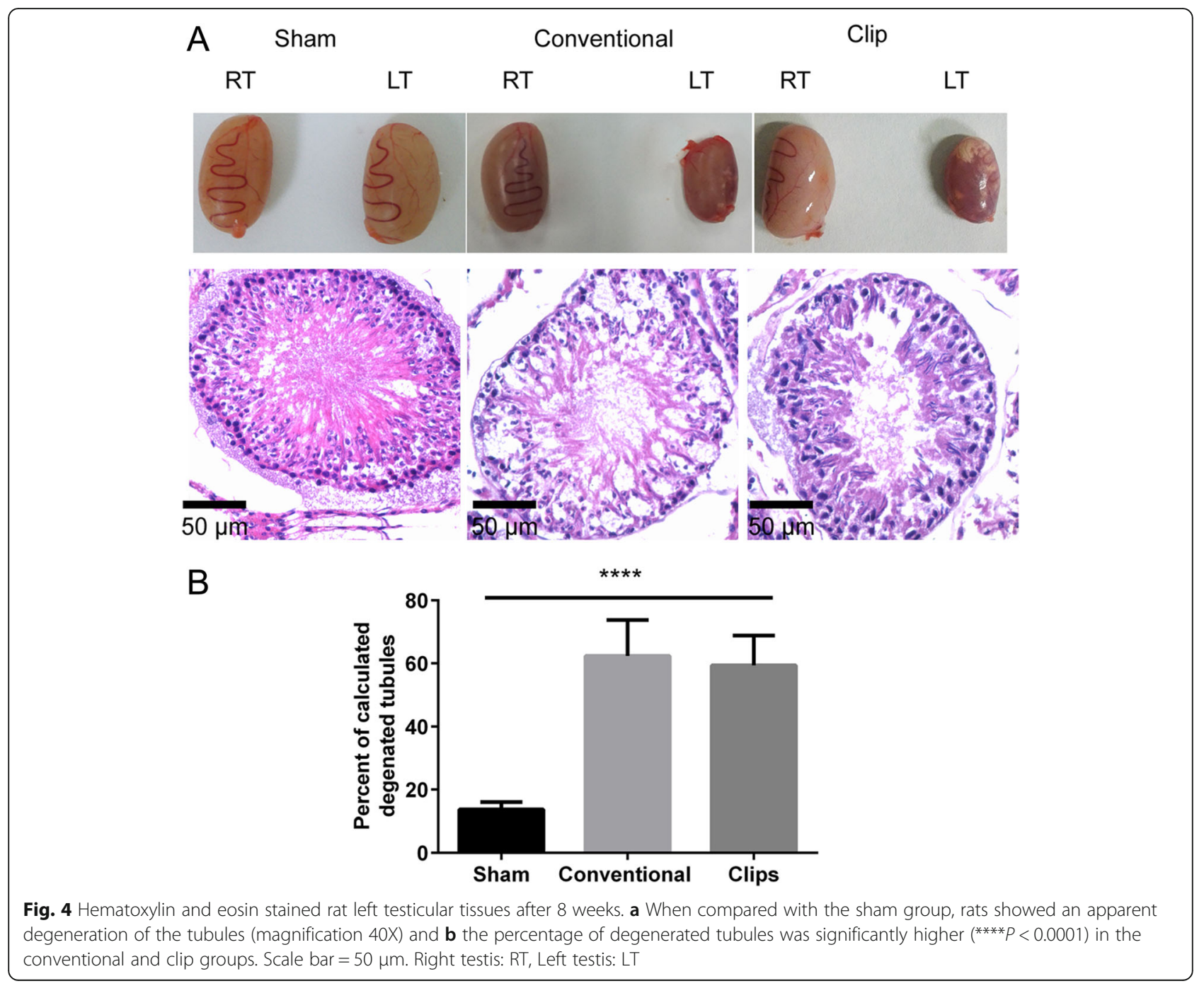

model by partially occluded the renal vein combined with completely ligation of the collaterals of the left spermatic vein with minor modifications. Furthermore, to improve the efficacy and create a consistent rat varicocele model, we used a novel clip technique usually applied to produce hypertension in the $2 \mathrm{~K} 1 \mathrm{C}$ model (2-kidney, 1-clip) [20]. The clip has more advantages of accurate and consistent reduction of renal vein diameter than suture ligation. To date, a key factor in current varicocele model is the potential for a correct and consistent degree of obstruction as a result of too much reduction in renal vein resulting necrosis or too little construction failing to form varicocele. Thus, clips with $0.7,0.8$ and $0.9 \mathrm{~mm}$ gap widths were selected in our study according to the $0.85 \mathrm{~mm}$ diameter as criteria reported by Turner TT [4]. At 8 weeks after initial operation, clip with $0.7 \mathrm{~mm}$ gap widths was appropriate and selected for further analysis in the study. No macroscopic changes and histological findings in the kidneys were observed among Group I, II and III, indicating there was no renal lesion paralleling the clinical findings of varicocele in humans [21]. The Group III had larger mean diameter of left spermatic vein and worse testicular histology than the Group I, similar to Group II but showing no significant difference. These results were accordance with these studies previously reported $[18,22]$.

Moreover, success rate of experimental varicocele rat model, up to a certain extent, depends on the complications during the operation [9]. In this study, the success rate in group 2 is only $75 \%(6 / 8)$. Many factors may account for this, including vascular injury, pyonephrosis. Sometimes when blinded to dissect behind the renal vein, inadvertent puncture or tear of the vein often occurs, resulting in excessive bleeding. Vascular injury also happens just when ligating the left renal vein. In Group II, 1 rat with pyonephrosis is mainly due to mistakenly ligation of ureter adjacent to spermatic vein, leading to obstructive hydronephrosis and infection. Using clip technique, we found no complications in Group III, and the success rate is $87.5 \%(7 / 8)$ except for 1 rat failing to form a varicocele because of one clip dislodging from 
the renal vein. Although the effects of clip technique on varicocele in this study, the convenient and safe model constitutes a basis for investigating on male infertility underlying varicocele, which is still further evaluated in subsequent studies that also need to expand the sample size.

There were also some inadequacies in this model. In Group IV and V, these clips were slipped off or dislodged from the renal vein, resulting in failing to form varicocele. The clip needs to be designed to eliminate the possibility of dislodgement after implantation with some modifications. In addition, although clip with $0.7 \mathrm{~mm}$ gap widths was appropriate in this model, the different diameters of clips should be further investigated in different characteristics of species, age, and weight in rat.

\section{Conclusion}

With a new clip technique, surgically inducing varicocele rat model becomes convenient and safe. This appears to improve the effectiveness of the model and this innovation may allow us to further understand the pathophysiology of varicocele.

\section{Abbreviations \\ 2K1C: 2-kidney, 1-clip; LSV: Left spermatic vein; PAS: Periodic acid-Schiff; SD: Standard deviation; SPF: Specific-pathogen free; VC: Varicocele}

\section{Acknowledgements}

The authors thank Dayu Han and Bing Yao for their contributions to the technique support of the model.

\section{Funding}

This study was supported in part by the Guangdong Provincial Science and Technology Program (No.2014A020212204; No.2013B021800317), Guangdong Provincial Natural Science Foundation of China (No.2015A030310027), Science and Technology Innovation Project of Southern Medical University (QD2014N005), Medical Scientific Research Foundation of Guangdong Province (A2014430) and National Natural Science Foundation of China (81772257).

\section{Availability of data and materials}

The datasets supporting the conclusions of this article are included within the article.

\section{Authors' contributions}

$\mathrm{CDL}$ and $\mathrm{XL}$ designed the study. QZZ, MKC and KYX carried out the experiments of animal surgery. WSZ and PW conducted the PAS and Masson's trichrome assays, HX and JKY participated in acquisition of data. CY and WBG analyzed the experimental data and drafted the manuscript. WBG also participated in revising the manuscript critically for important intellectual content and made substantial contributions to conception and design of the study. $\mathrm{XL}$ and JB participated in the revising of the manuscript. All authors read and approved the final manuscript.

\section{Ethics approval and consent to participate}

All experimental procedures were complied with the Guidelines of Animal Care of Southern Medical University, and were approved by the Ethics Committee of Southern Medical University, China. ARRIVE guidelines for reporting animal research was followed.

\section{Publisher's Note}

Springer Nature remains neutral with regard to jurisdictional claims in published maps and institutional affiliations.

Received: 5 May 2016 Accepted: 2 May 2018

Published online: 07 June 2018

\section{References}

1. Dubin L, Amelar RD. Etiologic factors in 1294 consecutive cases of male infertility. Fertil Steril. 1971;22(8):469-74.

2. Gorelick JI, Goldstein M. Loss of fertility in men with varicocele. Fertil Steril. 1993;59(3):613-6.

3. Naughton CK, Nangia AK, Agarwal A. Pathophysiology of varicoceles in male infertility. Hum Reprod Update. 2001;7(5):473-81.

4. Turner TT. The study of varicocele through the use of animal models. Hum Reprod Update. 2001;7(1):78-84.

5. Pascual JA, Lemmi C, Rajfer J. Variability of venous anatomy of rat testis: application to experimental testicular surgery. Microsurgery. 1992;13(6):335-7.

6. Hsu HS, Chang LS, Chen MT, Wei YH. Decreased blood flow and defective energy metabolism in the varicocele-bearing testicles of rats. Eur Urol. 1994;25(1):71-5

7. Li H, Dubocq F, Jiang Y, Tiquert R, Gheiler EL, Dhabuwala CB. Effect of surgically induced varicocele on testicular blood flow and Sertoli cell function. Urology. 1999;53(6):1258-62.

8. Yao B, Zhou WL, Han DY, Ouyang B, Chen X, Chen SF, Deng CH, Sun XZ. The effect of the degree of left renal vein constriction on the development of adolescent varicocele in Sprague-Dawley rats. Asian J Androl. 2016;18(3): 471.

9. Zhou T, Cao H, Chen G, Yang B, Sun Y. Outcomes of experimental rat varicocele with and without microsurgery. BMC Urol. 2015:15(1):19.

10. Tu L, Yu L, Zhang H. Morphology of rat testis preserved in three different fixatives. J Huazhong Univ Sci Technolog Med Sci. 2011;31(2):178-80.

11. RUSSELL JK. Varicocele in groups of fertile and subfertile males. Br Med J. 1954;1(4873):1231-3.

12. Saypol DC, Howards SS, Turner TT, Miller EJ. Influence of surgically induced varicocele on testicular blood flow, temperature, and histology in adult rats and dogs. J Clin Invest. 1981;68(1):39-45.

13. Rajfer J, Turner TT, Rivera F, Howards SS, Sikka SC. Inhibition of testicular testosterone biosynthesis following experimental varicocele in rats. Biol Reprod. 1987;36(4):933-7.

14. Barqawi A, Caruso A, Meacham RB. Experimental varicocele induces testicular germ cell apoptosis in the rat. J Urol. 2004;171(1):501-3.

15. Sahin Z, Celik-Ozenci C, Akkoyunlu G, Korgun ET, Acar N, Erdogru T, Demir R, Ustunel I. Increased expression of interleukin-1alpha and interleukin-1beta is associated with experimental varicocele. Fertil Steril. 2006;85(Suppl 1): 1265-75.

16. Turner TT, Howards SS. The venous anatomy of experimental left varicocele: comparison with naturally occurring left varicocele in the human Fertil Steril. 1994;62(4):869-75.

17. Turner TT, Caplis LA, Brown KJ. Vascular anatomy of experimentally induced left varicocele in the rat. Lab Anim Sci. 1996;46(2):206-10.

18. Zhang LT, Kim HK, Choi BR, Zhao C, Lee SW, Jang KY, Park JK. Analysis of testicular-internal spermatic vein variation and the recreation of varicocoele in a Sprague-Dawley rat model. Andrology. 2014;2(3):466-73.

19. Lewis MH, Moffat DB. The venous drainage of the accessory reproductive organs of the rat with special reference to prostatic metabolism. J Reprod Fertil. 1975:42(3):497-502.

20. Dussaule JC, Michel JB, Auzan C, Schwartz K, Corvol P, Menard J. Effect of antihypertensive treatment on the left ventricular isomyosin profile in oneclip, two kidney hypertensive rats. J Pharmacol Exp Ther. 1986;236(2):512-8.

21. Takemura T, Iwasa H, Yamamoto S, Hino S, Fukushima K, Isokawa S, Okada M, Yoshioka K. Clinical and radiological features in four adolescents with nutcracker syndrome. Pediatr Nephrol. 2000;14(10-11):1002-5.

22. Yao B, Zhou W, Han D, Ouyang B, Chen X, Chen S, Deng C, Sun X. The effect of the degree of left renal vein constriction on the development of adolescent varicocele in Sprague-Dawley rats. Asian J Androl. 2016;18(3):471.

\section{Competing interests}

The authors declare that they have no competing interests. 